\title{
The Role of Low Dose CT in Diagnosis of Uretric Stones
}

\author{
Firas Salim Daoud Karomy \\ High Diploma in Diagnostic Radiology/ College of Medicine/ University of Baghdad
}

\begin{abstract}
Urolithiasis is a common disease worldwide and affects a wide gamut of the patient population, irrespective of race, culture or geographic boundaries. In the past few decades, there has been an increasing incidence of urinary stone disease both in developed and developing nations due to changes in life style, particularly due to the rising prevalence of obesity. MDCT plays a critical role in the management of patients with urolithiasis. Their applicability ranges from the initial diagnosis to planning treatment strategies and in post-treatment follow-up. The emerging technological innovations in CT are bridging the gap between radiological interpretations and urologists' expectations. With the widespread implementation of low-dose protocols and iterative reconstruction algorithms in routine clinical practice, the radiation dose concerns can be minimized without affecting the diagnostic yield of the CT exams.
\end{abstract}

Keywords: urolithiasis, implementation, urologists, algorithms, urinary stone

\section{Introduction}

Urolithiasis is a common disease worldwide and affects a wide gamut of the patient population, irrespective of race, culture or geographic boundaries. In the past few decades, there has been an increasing incidence of urinary stone disease both in developed and developing nations due to changes in life style, particularly due to the rising prevalence of obesity. ${ }^{[1,2,3]}$ Recent studies have also demonstrated a changing composition of urolithiasis as well as an appreciable increase in the incidence of stone disease in females and younger patients over the last decade. ${ }^{[1,4]}$ Appropriate management of urolithiasis has important clinical implications due to their association with complications such as infection and chronic kidney disease and high rate of recurrence. ${ }^{[1,3]}$

Imaging has a critical role in the initial diagnosis, treatment planning and post-treatment surveillance of patients with urolithiasis. Unenhanced computed tomography (CT), first introduced for stone imaging in the 1990s, has since emerged as the reference gold standard for the initial and subsequent evaluation of patients with suspected kidney stones, superseding radiography and intravenous urography. Non-contrast CT offers several advantages compared with alternative imaging techniques such as plain radiography and ultrasound, including high sensitivity and specificity ( $>95 \%$ and $>96 \%$, respectively) for the detection of stones, easy availability, faster speed of acquisition and absence of need for administration of intravenous contrast. ${ }^{[5,6,7]}$ With the emergence of multi-detector CT (MDCT) and advanced technologies like dualenergy CT (DECT), the scope of CT in urolithiasis management has further expanded. Besides aiding in accurate diagnosis, MDCT also plays an important role in treatment planning, follow-up and assessment of treatment success due to its ability to characterize stone composition and fragility ${ }^{[8,9]}$ In this review article, we will discuss the current and emerging role of CT in the management of patients with urinary stone disease and their impact on planning treatment strategies and patient follow-up. The use of bone window settings is helpful in these instances to better differentiate between the stones and stents, which can appear to have similar density on soft tissue window settings. 


\section{MDCT}

Unenhanced helical CT is highly sensitive (up to 98\%) and specific (96-100\%) in diagnosing urolithiasis, and is the imaging modality of choice for the initial evaluation of patients with suspected urinary stones. $[5,6] \mathrm{CT}$ is highly preferred due to easy availability, speed, ease of image acquisition, absence of need for oral or intravenous contrast media administration, and ability to detect extra-urinary pathologies such as appendicitis, diverticulitis or gynecological pathologies such as hemorrhagic cyst or ovarian torsion that may mimic the renal colic. ${ }^{[5,6,10,11]}$ This is especially crucial in managing patients presenting with acute abdominal pain imitating renal colic in emergency departments (EDs) because, often times, these patients are not evaluated by urologists and therefore excluding other potential abdominal pathologies are also concurrent objectives of the ordering physicians. ${ }^{[5,12]}$ In patients presenting to the emergency room with symptoms suggestive of renal colic, $\mathrm{CT}$ enables an alternative diagnosis in nearly 10-14\%. ${ }^{[13,14,15,16,17]}$ Furthermore, CT also permits the diagnosis of urinary abnormalities such as congenital conditions of the urinary tract and renal/urothelial neoplasms, recognition of which has immense clinical implications to patient management and prognosis.

MDCT, introduced in 1998, has opened new opportunities in the management of urolithiasis, such as the ability to perform multi-planar reformations and three-dimensional (3D) reconstructions that have enhanced the detection and quantification of urinary stone burden. Besides routine evaluation of the number of stones, location, size and presence or absence of hydronephrosis, the volumetric analysis of stone burden by MDCT has been a strong indicator for treatment planning and outcome. Furthermore, MDCT allows assessment of stone composition by measuring attenuation in Hounsfield Units (HU). ${ }^{[18]}$

\section{MDCT technique}

Stone protocol CT tailored for the diagnosis of urinary stone disease varies from a routine non-contrast abdomino-pelvic CT study and has different scan acquisition parameters. The coverage area for a stone protocol CT extends from the upper pole of both kidneys to the base of the urinary bladder. ${ }^{[14,22,23,24]}$ Although thinner slices $(1-3 \mathrm{~mm})$ are desirable, acquiring CT images at a slice thickness of $5 \mathrm{~mm}$ complimented with 3 $\mathrm{mm}$ coronal/sagittal reformatted images improves stone detection while lowering radiation dose.[23,25] A tube potential of $100-120 \mathrm{kVp}$ and automatic tube current modulation (ATCM) with an $\mathrm{mA}$ range of $80-500 \mathrm{~mA}$ is frequently used; however, it should be noted that the scan acquisition protocols are tailored to the patient body weight and CT scanner technology.[26] With the introduction of iterative reconstruction (IR) technology, stone protocol CT scans can be performed at lower mA and lower $\mathrm{kVp}$, permitting substantial radiation dose reduction. The $\mathrm{CT}$ acquisition parameters for suspected renal stone evaluation in the emergency department scanners are slightly different from the routine stone protocol CTs to improve the ability of ED physicians in ruling out other potential non-urinary causes of patient symptoms. This includes acquiring thinner slices and adapting a scan coverage length similar to the abdomino-pelvic CT scan. Contrast administration is not required for routine stone diagnosis; however, it may be of value in identifying vascular calcifications or for differentiating distal ureteral stones from phlebolithsThe use of intravenous contrast should also be reserved for equivocal cases or in instances where possibilities of alternate diagnosis are high.

Coronal and sagittal reformatted images of $3 \mathrm{~mm}$ thickness are routinely acquired and are an indispensable part of the stone CT protocol. Integration of multi-planar reformatted images with routine axial scans during image interpretation enables precise evaluation of the entire urinary tract and location of the impacted stones. They also improve the detection of small stones, particularly at the renal poles, and facilitate the differentiation of extrarenal calcifications from urinary stones.[13]

\section{CT detection of Urolithiasis}

Evaluation of urolithiasis using MDCT should include interpretation of both axial and multi-planar 
reformatted images to improve accuracy. Unenhanced CT detects all types of urinary tract calculi, including stones such as uric acid, xanthine or cystine stones that are otherwise radiolucent on conventional radiographs. [5] The only exceptions are pure matrix stones and stone encountered in patients on indinavir treatment, which are usually missed on MDCT due to their soft tissue attenuation $(15-30 \mathrm{HU}) .{ }^{[5,14]}$ In these situations, administration of intravenous contrast and delayed imaging might facilitate their diagnosis. ${ }^{[14]}$

The stone location and its site of impaction in the ureter are very important in stone management, with the treatment success rates being better for calculi located in the lower third of the ureter. Compared with conventional radiography, CT enables precise detection and localization of ureteral calculi: ${ }^{[15]}$ Direct visualization of stone in the ureteric lumen with proximal ureteral dilatation and normal caliber of the distal ureter is a common finding. ${ }^{[7]}$ The absence of ureteral dilatation has however been reported in a small number of cases, and presence or absence of ureteral dilatation does not indicate presence or absence of urinary tract obstruction. Certain secondary signs aid in the diagnosis of ureteral stones on $\mathrm{CT}$, including reliable signs such as perinephric fat stranding, periureteral edema, hydroureter and hydronephrosis and less-consistent signs such as perinephric edema and lateral conalfascial thickening. The positive and negative predictive values of intra-renal collecting system dilatation and perinephric fat stranding in detecting ureterolithiasis nears 98\% and 91\%, respectively.[5,16] The failure to detect stone in patients with a high degree clinical suspicion necessitates a repeat meticulous evaluation for secondary signs. The presence of ureteric dilatation and perinephric stranding makes ureterolithiasis more likely, and the possible scenarios include either passage of a previously obstructing stone or presence of a stone with size or attenuation features, which limit detection on MDCT. On the contrary, the absence of these secondary signs allows one to reliably rule out urinary stone disease and necessitates evaluation of other potential causes of patient symptoms, including extra-urinary pathologies that mimic ureteric colic. ${ }^{[5]}$

\section{CT in the assessment of stone burden}

A key element in the urological management of stone disease, which can consistently and significantly impact decision making, is the estimation of stone burden ${ }^{[15,17]}$ Stone size is a simpler metric for stone burden assessment and can be reliably obtained on CT. Urological decisions on the selection of treatment strategies, including need of medical expulsive therapy or endoscopic/percutaneous interventions, rely strongly on stone size determination. Furthermore, stone size also helps in deciding on the type of urological interventions, e.g., shock wave lithotripsy (SWL) versus ureteroscopy with lithotripsy versus percutaneous nephrolithotomy (PCNL). Stone size can be reliably estimated on CT by measuring the largest dimension. Measurements can be made on the soft tissue window (window width -400 $\mathrm{HU}$ and window level $-30 \mathrm{HU}$ ) or on a bone window (window width $-1120 \mathrm{HU}$ and window level-300 HU). $[28,29]$

\section{Stone volumetry}

Despite the ease of using two-dimensional (2-D) measurements for estimation of stone burden, they are restricted in their ability to accurately quantify stone size for large stones with irregular contour such as stag horn calculi. Stone volumetry, a technique that allows estimation of stone volume, counteracts this limitation and allows accurate assessment of stone burden with MDCT. Different methods have been employed to determine the stone volume, including obtaining the product of three orthogonal measurements, generation of 3D volume measurements from the stone circumference data on various stone-bearing image sets and, more recently used, semi-automatic segmentation tools ${ }^{[18]}$ Stone volume, besides being a valuable tool for pre-operative planning, has also been shown to be a reliable predictor of treatment success ${ }^{[18]}$ For example, it has been shown that using a stone volume cut-off of $700 \mathrm{~mm} 3$ can be used to successfully predict treatment outcome in patients undergoing SWL. 


\section{CT in the determination of stone fragility}

Stone internal structure assessment is another imaging determinant that can impact the outcome following urological interventions, especially after SWL. High-resolution MDCT scanning with thin slices and reconstruction with bone algorithm allows visualization of the internal structure/architecture of the stones, particularly when seen on bone window setting. The internal architecture as seen on CT could either suggest internal homogeneity or heterogeneity. Stones with internal homogeneity have a uniform internal structure, are more rigid and are difficult to break with lithotripsy. On the other hand, stones with internal heterogeneity have areas of low attenuation or internal voids within the stone component. The internal heterogeneity is an indication of high stone fragility and the internal irregularities within-stone structures facilitate easy disintegration of stones on SWL. ${ }^{[9]}$ Even for the inherently hard brushite, cystine and calcium oxalate monohydrate stones, presence of heterogeneity has been shown to increase the success of fragmentation with SWL ${ }^{[13]}$ On the contrary, stones with internal homogeneity are resistant to easy fragmentation and often necessitate multiple therapeutic sessions.

\section{CT in the determination of stone composition}

Urological management of urinary tract calculi relies on several different factors such as stone size, location, number, anatomy and chemical composition. Precise pre-treatment determination of urinary stone composition is essential and considerably impacts appropriate management. ${ }^{[6]}$ For instance, uric acid stones are suitable for medical management with oral medications that assist in stone dissolution. While struvite stones are sensitive to SWL, cysteine stones and calcium oxalate monohydrate stones are comparatively resistant to treatment by SWL ${ }^{[5]}$ Knowledge of the stone composition is also useful for the prevention of recurrent disease. Traditionally, patient history, urine $\mathrm{pH}$, urinary crystals, urease-positive organisms and plain radiographs were employed to predict stone composition. ${ }^{[4]}$ More recently, $\mathrm{CT}$ is being increasingly used for the in vivo determination of stone composition and the emergence of new technological innovations such as DECT permits reliable determination of stone composition. ${ }^{[3]}$

\section{Region of interest based methods}

The most traditional method for estimation of stone composition on MDCT has been using placement of the ROI over the stone to obtain the attenuation value in $\mathrm{HU}$. Bellin and colleagues showed that HU measurements based on ROI placement had an accuracy of $64-81 \%$ in predicting the composition of urinary stones in in vitro studies ${ }^{[38]}$ Similar studies have shown that HU measurements obtained using CT can be used to reliably identify uric acid, cysteine and calcium oxalate monohydrate stones with considerable accuracy $(>85 \%)$ in in vitro studies ${ }^{[2]}$ The HU measurements of the various urinary stones at $120 \mathrm{kV}$ usually fall under the following range: Uric acid, 200-450 HU; struvite, 600-900 HU; cysteine, 600-1100 HU; calcium phosphate, 1200-1600 $\mathrm{HU}$; and calcium oxalate monohydrate and brushite, 1700-2800 HU. ${ }^{[8]}$ Despite the substantial accuracy of $\mathrm{CT}$ in determining stone composition in in vitro studies, HU measurement for predicting stone composition in vivo is less reliable and challenging. Stone composition assessment using attenuation values (HU) is often dependent on the size of the ROI, slice thickness and accurate placement of ROI over the stone to eliminate partial volume averaging effects ${ }^{[5]}$ Most often, the stones encountered in vivo are of mixed composition, which makes precise determination complex, and furthermore the overlap in attenuation measurements of various stones have limited the role of ROI-based methods in distinguishing different type of stones. ${ }^{[1]}$

\section{DECT}

DECT technology introduced in the past decade holds great promise for the accurate determination of stone composition ${ }^{[46,47,48,49]}$ Dual-energy scanning involves simultaneous scanning using two different energies, which permits tissue characterization. Two different DECT systems are currently available in clinical practice: Dual-source DECT (dsDECT) and single-source DECT (ssDECT). DsDECT is assembled 
with two X-ray tubes (140 and $80 \mathrm{kVp}$ ) and two detectors disposed in a single gantry perpendicular to each other and ssDECT is assembled with one $\mathrm{X}$-ray tube with rapid $\mathrm{kV}$ switching between high $(140 \mathrm{kVp})$ and low energy $(80 \mathrm{kVp})$.[50] Along with the distinct hardware configurations, these systems have different postprocessing techniques that allow material separation and generation of images with different $\mathrm{X}$-ray energies $(\mathrm{keV})$.

Besides its high sensitivity for the detection of urolithiasis, DECT offers enhanced ability to characterize stone composition and differentiate between various stone types. Determination of stone composition has been one of the most explored and recognized applications of DECT in the abdomen, and this technology has been validated in vivo and in vitro studies using both dsDECT and ssDECT, particularly for differentiation of uric acid and non-uric acid stones. ${ }^{[46,48]}$ DECT allows determination of stone composition based on the principle of variation in attenuation characteristics of stones at different X-ray energies based on their composition. DECT allows differentiation of uric acid and non-uric acid (calcium-dominant) stones based on the fact that the uric acid stones are composed of elements with low atomic numbers $(\mathrm{H}, \mathrm{C}, \mathrm{N}, \mathrm{O})$ and their X-ray attenuation profile at multiple energies is different compared with that of non-uric acid stones, which are composed of elements with higher atomic numbers (P, Ca, S). ${ }^{[6]}$ Based on this differential behavior, the dsDECT algorithm assumes that every voxel is a mixture of water, calcium and uric acid. Voxels with $\mathrm{X}$-ray attenuation profile similar to calcium are colored distinctly from those with an attenuation profile similar to uric acid ${ }^{[12]}$ In contrast, CT data acquired from an ssDECT scan is processed using a two-material (basis pair) decomposition algorithm on the scanner console generating two image series, generally iodine and water (high and low atomic number materials, respectively). Stones visualized on water images only are considered uric acid stones, whereas those stones visualized on both water and iodine images are characterized as non-uric acid calculi. Material decomposition images have shown
$100 \%$ sensitivity and accuracy in distinguishing uric acid and non-uric acid stones, regardless of the size of the stones ${ }^{[14]}$ Additionally, effective Z (Zeff) images can be generated in vendor-specific workstations (ADW version 4.5; GE Healthcare, Milwaukee, WI, USA) for further analysis of renal stone composition. Zeff is a validated method for renal stone characterization and facilitates the identification of predominant material within mixed stones by taking into account the attenuation and atomic number of a specific material. Low Zeff is suggestive of uric acid stones, whereas high Zeff suggests non-uric acid stones. Accurate sub-categorization of renal stones is an evolving application of DECT and, with evolving DECT algorithms further subtype differentiation is becoming available. For instance, a tin filter installed to the high-energy tube of new-generation dsDECT scanners decreases the overlap between the two energy potentials, improving the spectral separation, which allows further characterization of renal stones with similar composition.[10]

Characterization of kidney stones using dualenergy computed tomography (DECT). DECT helps to distinguish between uric acid and non-uric acid renal stones. a and c are axial images acquired from different patients who presented with flank pain. (a) Axial image showing a non-uric acid renal stone in the right kidney (arrowhead) colored in blue. (b) Graph showing the composition of this stone (blue arrowhead). (c) Axial image showing a uric acid renal calculus in the left kidney (arrow) colored in red. (d) Graph confirms the composition of the stone (red arrowhead)

The current DECT protocol for renal stones includes a low-dose MDCT acquisition using the single-energy mode, covering the abdomen and pelvis, to identify possible calculi in the urinary tract. Once the urinary stone is localized, a dual-energy acquisition targeted to the anatomical area of the stone is performed to minimize the total radiation dose.[14] After scan acquisition, the DECT data are processed on the CT console, generating different images series. Images with a specific virtual X-ray energy (50-70 keV) are generated for improved visualization of the renal stones by enhancing the contrast 
differences with the surrounding tissues. Because each DECT acquisition produces more datasets, the number of images and total reconstruction time for DECT studies is longer compared with single energy CT (SECT) studies. A vendor-specific workstation is required to generate Zeff images from DECT scans. The resultant DECT images from the reconstruction process can be viewed the same way as the SE-CT images.

Characterization of mixed renal stones using DECT can be challenging; however, the current technology allows recognition of the predominant stone type. Reported shortcomings of first-generation dsDECT for urolithiasis include a decrease in specificity for stones $<3 \mathrm{~mm}$ and in patients with large body habitus. ${ }^{[15]}$ However, these small stones are spontaneously passed, having less clinical relevance.

\section{CT in planning percutaneous interventions}

In addition to contributing to treatment planning by determination of stone burden, location, composition and fragility, MDCT also plays an important role in the pre-surgical evaluation of patients who are candidates for interventional procedures like PCNL.[14] MDCT aids in the evaluation of the position of kidneys, the orientation of the pyelocalyceal system and the relationship of the kidney to various surrounding organs like spleen, liver and colon ${ }^{[17]}$

Various parameters crucial for successful calyceal access during PCNL, including localization of posterior calyx and angle between the calyces, can be reliably evaluated using MDCT. The multi-planar reformations and 3-D post-processing have made visualization of the calyceal system precise and easy. Similarly, for patients who are candidates for SWL, stone-to-skin distance (SSD) is an important predictor for stone-free survival. $\mathrm{SSD}$, which is the distance from the center of the stone to the skin surface, can be reliably determined on the axial MDCT scans[2] An SSD greater than $10 \mathrm{~cm}$ on $\mathrm{CT}$ has been shown to increase the failure rate of SWL and therefore PCNL or other ureteroscopic interventions may be advocated in these patients. ${ }^{[4]}$
Measurement of stone-to-skin distance (SSD) in a 54-year-old man. On an axial non-contrast computed tomography scan, the distance from the center of the stone to the surface of the skin at $0^{\circ}, 45^{\circ}$ and $90^{\circ}$ is 8.0 , 8.7 and $9.4 \mathrm{~cm}$, respectively. The mean of these three measurements is used to represent the average SSD, which is $8.7 \mathrm{~cm}$

\section{CT in post-treatment follow-up}

Following urological treatment of patients with stone disease, the main objectives of CT imaging are to (i) ascertain stone-free status, (ii) identify the presence of residual stones, (iii) rule out stricture in the urinary system and (iv) detect any complications related to urological interventions. ${ }^{[15,18]}$ MDCT is a modality of choice for identifying residual stone burden after interventional procedures like PCNL and SWL. CT aids in accurately localizing the residual fragments in the kidney/ureters and thereby facilitates their removal. This is essential because recurrence rates are higher in patients (50-80\%) with persistent residual stones compared with those with stone-free status. CT has a definitive role in the follow-up of stones that are lucent on conventional imaging; however, its additional value in stones that are radioopaque on KUB or scout images remains debatable ${ }^{[13]}$

\section{Stone versus stent}

Following urologic interventions, ureteral stents or nephrostomy tubes are often placed during the immediate post-treatment period to facilitate urine drainage. In these patients, it is essential to differentiate residual stone fragments in the renal collecting system or ureters from in situ stents for appropriate post-treatment follow-up. The use of bone window settings is helpful in these instances to better differentiate between the stones and stents, which can appear to have similar density on soft tissue window settings

An external file that holds a picture, illustration, etc.

Staghorn calculus in a 47-year-old man with treated lithotripsy. (a) Axial non-contrast computed 
tomography (CT) obtained prior to lithotripsy shows large right staghorn calculus with hydronephrosis of the upper pole renal calyces. (b) Post-treatment CT scan in soft tissue window settings (400/30) shows reduction in stone burden with residual stone fragments in the renal pelvis. (c) $\mathrm{CT}$ image in the bone window settings (1100/300) allows improved distinction of the stone from the ureteral stent

\section{CT in urolithiasis and ionizing radiation}

Ionizing radiation exposure from $\mathrm{CT}$ scans and its harmful effects remains a concern for healthcare professionals and patients alike. In patients with urolithiasis, it is particularly concerning for young patients with recurrent disease who need to undergo repeated $\mathrm{CT}$ scans and are therefore at increased risk of cumulative radiation exposure ${ }^{[18]}$ The reported cumulative radiation dose associated with repeated CT exams may range from 8.5 to as high as $154 \mathrm{mSv}^{\left[{ }^{[16]}\right.}$ A crucial step in limiting radiation exposure is through judicious use of $\mathrm{CT}$ in evaluation of patients with stone disease. For example, in pregnant patients and children, ultrasound could be favored for the diagnosis of stone disease ${ }^{[13]}$

\section{Conclusion}

MDCT plays a critical role in the management of patients with urolithiasis. Their applicability ranges from the initial diagnosis to planning treatment strategies and in post-treatment follow-up. The emerging technological innovations in $\mathrm{CT}$ are bridging the gap between radiological interpretations and urologists' expectations. With the widespread implementation of low-dose protocols and iterative reconstruction algorithms in routine clinical practice, the radiation dose concerns can be minimized without affecting the diagnostic yield of the CT exams.

Conflict of Interest: There is no conflict of interest among the authors.

Funding: Self

Ethical Clearance: This study is ethically approved by the Institutional ethical Committee.

\section{References}

1. Neisius A, Preminger GM. Stones in 2012: Epidemiology, prevention and redefining therapeutic standards. Nat Rev Urol. 2013;10:75-7. [PubMed] [Google Scholar]

2. Bartoletti R, Cai T, Mondaini N, Melone F, Travaglini F, Carini M, et al. Epidemiology and risk factors in urolithiasis. Urol Int. 2007;79(Suppl 1):3-7. [PubMed] [Google Scholar]

3. Scales CD, Jr, Smith AC, Hanley JM, Saigal CS. Urologic Diseases in America Project. Prevalence of kidney stones in the United States. Eur Urol. 2012;62:160-5. [PMC free article] [PubMed] [Google Scholar]

4. Seitz C, Fajkovic H. Epidemiological genderspecific aspects in urolithiasis. World $\mathrm{J}$ Urol. 2013;31:1087-92. [PubMed] [Google Scholar]

5. Smith RC, Verga M, McCarthy S, Rosenfield AT. Diagnosis of acute flank pain: Value of unenhanced helical CT. AJR Am J Roentgenol. 1996;166:97101. [PubMed] [Google Scholar]

6. Dhar M, Denstedt JD. Imaging in diagnosis, treatment, and follow-up of stone patients. Adv Chronic Kidney Dis. 2009;16:39-47. [PubMed] [Google Scholar]

7. Pfister SA, Deckart A, Laschke S, Dellas S, Otto U, Buitrago C, et al. Unenhanced helical computed tomography vs intravenous urography in patients with acute flank pain: Accuracy and economic impact in a randomized prospective trial. EurRadiol. 2003;13:2513-20. [PubMed] [Google Scholar]

8. Rosen MP, Siewert B, Sands DZ, Bromberg R, Edlow J, Raptopoulos V. Value of abdominal CT in the emergency department for patients with abdominal pain. EurRadiol. 2003;13:418-24. [PubMed] [Google Scholar]

9. Kaza RK, Platt JF, Cohan RH, Caoili EM, AlHawary MM, Wasnik A. Dual-energy CT with single- and dual-source scanners: Current applications in evaluating the genitourinary tract. Radiographics. 2012;32:353-69. [PubMed] [Google Scholar]

10. Boulay I, Holtz P, Foley WD, White B, Begun FP. Ureteral calculi: Diagnostic efficacy of helical CT and implications for treatment of patients. AJR Am J Roentgenol. 1999;172:1485-90. [PubMed] 
[Google Scholar]

11. Katz DS, Lane MJ, Sommer FG. Unenhanced helical CT of ureteral stones: Incidence of associated urinary tract findings. AJR Am J Roentgenol. 1996;166:1319-22. [PubMed] [Google Scholar]

12. Carter MR, Green BR. Renal calculi: Emergency department diagnosis and treatment. Emerg Med Pract. 2011;13:1-17. quiz 8. [PubMed] [Google Scholar]

13. Metser U, Ghai S, Ong YY, Lockwood G, Radomski SB. Assessment of urinary tract calculi with 64MDCT: The axial versus coronal plane. AJR Am J Roentgenol. 2009;192:1509-13. [PubMed] [Google Scholar]

14. Schwartz BF, Schenkman N, Armenakas NA, Stoller ML. Imaging characteristics of indinavir calculi. J Urol. 1999;161:1085-7. [PubMed] [Google Scholar]
15. Eisner BH, McQuaid JW, Hyams E, Matlaga BR. Nephrolithiasis: What surgeons need to know. AJR Am J Roentgenol. 2011;196:1274-8. [PubMed] [Google Scholar]

16. Ege G, Akman H, Kuzucu K, Yildiz S. Acute ureterolithiasis: Incidence of secondary signs on unenhanced helical $\mathrm{CT}$ and influence on patient management. ClinRadiol. 2003;58:990-4. [PubMed] [Google Scholar]

17. Preminger GM, Assimos DG, Lingeman JE, Nakada SY, Pearle MS, Wolf JS., Jr Chapter 1: AUA guideline on management of staghorn calculi: Diagnosis and treatment recommendations. J Urol. 2005;173:1991-2000. [PubMed] [Google Scholar]

18. Bandi G, Meiners RJ, Pickhardt PJ, Nakada SY. Stone measurement by volumetric threedimensional computed tomography for predicting the outcome after extracorporeal shock wave lithotripsy. BJU Int. 2009;103:524-8. [PubMed] [Google Scholar] 